\title{
Colitis actínica de localización cecal. Serie de casos
}

\section{Actinic colitis of cecal location. Number of cases}

María M. Rojas-Rojas, MD, ${ }^{1}$ Catalina Buriticá-Cifuentes, MD, ${ }^{2}$ Luis E. Barrera-Herrera, MD, ${ }^{1}$ Marcela Mejía-Arango, MD, ${ }^{2}$

David A. Suárez-Zamora, MD, ${ }^{1}$ Johanna Álvarez-Figueroa, MD. ${ }^{*}$

Departamento de Patología y Laboratorios, Fundación Santa Fe de Bogotá. Bogotá D. C., Colombia.

Departamento de Patología y Laboratorios, Fundación Santa Fe de Bogotá. Facultad de Medicina, Universidad de los Andes. Bogotá D. C., Colombia.

*Correspondencia: Johanna Álvarez-Figueroa, MD johanna.alvarez@fsfb.org.co

Fecha recibido: $12 / 02 / 19$

Fecha aceptado: $30 / 04 / 19$

\section{Resumen}

Por definición, la colitis actínica incluye cambios inflamatorios de la mucosa colorrectal secundarios a radioterapia en cercanía a la región tratada. La localización más frecuente es el recto y la indicación más común de radioterapia corresponde a neoplasias de la región pélvica incluidos el recto, la próstata y el cérvix. Se estima que hasta la mitad de los pacientes que reciben radiación pélvica llega a desarrollar síntomas gastrointestinales asociados. Se presentan dos pacientes con metástasis óseas sacroilíacas y pélvicas secundarias a adenocarcinoma de próstata que recibieron radiación en la región lumbosacra y pélvica, ambos pacientes presentaron episodios de deposiciones con sangre que iniciaron tempranamente posterior a la radioterapia. La colonoscopia mostró eritema y ulceración. En el estudio histopatológico se observó un patrón de colitis isquémica, con núcleo y citomegalia, estroma fibroso con cambios reactivos y abundante infiltrado inflamatorio neutrofílico. Estos hallazgos son característicos de la colitis actínica aguda; sin embargo, la localización cecal no ha sido frecuentemente reportada. Aunque al ser el ciego y el íleon terminal móviles de localización pélvica, se convierten en un factor de riesgo para que estos segmentos anatómicos sean susceptibles al impacto directo de la radioterapia. Esta condición, en la fase aguda, es autolimitada y se suele resolver con medidas de soporte. Es indispensable que el personal involucrado en el manejo de estos pacientes conozca esta entidad y los posibles diagnósticos diferenciales.

\section{Palabras clave}

Colitis, radioterapia, hemorragia gastrointestinal.

\begin{abstract}
By definition, actinic colitis includes inflammatory changes of the colorectal mucosa secondary to radiation therapy of nearby tissue. The most frequent location is the rectum, and the most common indication for radiation therapy is a pelvic region neoplasm in the rectum, prostate or cervix. It is estimated that up to half of patients receiving pelvic radiation go on to develop associated gastrointestinal symptoms.

We present two patients with sacroiliac and pelvic bone metastases secondary to prostate adenocarcinoma who received radiation in the lumbosacral and pelvic region. Both patients developed bloody stools soon after radiation therapy. Colonoscopy showed erythema and ulceration, and histopathology found a pattern of ischemic colitis with nucleus and cytomegalovirus infection, fibrous stroma with reactive changes and abundant inflammatory infiltration of neutrophils. These findings are characteristic of acute actinic colitis, but the cecal location has not been reported frequently. Nevertheless, the pelvic location of the cecum and the terminal ileum puts these anatomical segments at risk from the direct impact of radiation therapy. In the acute phase, this condition, is self-limiting and usually resolves with support measures. It is essential that the personnel involved in the management of these patients be aware of this entity and its possible differential diagnoses.
\end{abstract}

\section{Keywords}

Colitis, radiotherapy, gastrointestinal bleeding. 


\section{INTRODUCCIÓN}

La colitis actínica o por radioterapia corresponde a cambios inflamatorios de la mucosa del colon secundarios a radioterapia en cercanía de la región afectada (1). Corresponde a una patología frecuente que se presenta en hasta aproximadamente un $50 \%$ de los pacientes que reciben tratamiento de radioterapia para neoplasias malignas abdominales y pélvicas. Usualmente, los pacientes con cáncer de próstata, cérvix y recto son los más afectados y las regiones que suelen estar comprometidas son el colon sigmoide y el recto (2, 3). La colitis actínica puede ser aguda o crónica, de acuerdo con el tiempo de aparición de la lesión con respecto a la exposición: la fase aguda se presenta en los primeros seis meses posteriores al inicio de la radioterapia y suele manifestarse clínicamente con diarrea, tenesmo y sangrado; la fase crónica se presenta después de seis meses de la exposición y se presenta con diarrea, dolor, obstrucción, fístulas y sangrado. El diagnóstico convencionalmente involucra los hallazgos de la colonoscopia y la historia clínica (3). La colitis por radioterapia localizada en el ciego es infrecuente y no se encuentran casos reportados en la literatura. Se presentan dos casos de colitis actínica de localización cecal en pacientes irradiados por metástasis pélvicas óseas de adenocarcinoma de próstata.

\section{CASO 1}

Un paciente de 75 años con antecedente de adenocarcinoma de próstata consulta por un cuadro de dolor en la cadera derecha. Se realizó una tomografía axial computarizada
(TAC) de abdomen que evidenció múltiples lesiones osteoblásticas en la columna, pelvis y fémur secundarias, con un probable compromiso metastásico. Se indicó una radioterapia con intención paliativa (300 cGy por sesión) en toda la región pélvica que incluyó las cabezas femorales. Al terminar 10 sesiones de radioterapia el paciente presentó deposiciones con sangre. Se realizó una colonoscopia que mostró mucosa del ciego con leve eritema focal sin úlceras ni otras lesiones y se tomaron biopsias en esta localización. El estudio histopatológico (Figura 1) mostró un patrón histológico de colitis isquémica aguda con cambios reactivos, pérdida y miniaturización de glándulas, citomegalia y nucleomegalia sin alteración de la relación núcleo-citoplasma, lámina propia paucicelular con infiltrado inflamatorio de predominio neutrofílico y superficial con fibrosis incipiente, edema y zonas con hemorragia, sin trombos. Con estos hallazgos se diagnosticó una colitis actínica aguda y el paciente recibió tratamiento con medidas de soporte, en el cual presentó una adecuada evolución.

\section{CASO 2}

Un paciente de 85 años con diagnóstico de adenocarcinoma de próstata metastásico en la región lumbar y sacroilíaca derecha consultó un por cuadro de melenas y hematoquecia que iniciaron siete días después de finalizar seis sesiones de radioterapia en la región lumbosacroilíaca derecha ( 400 cGy por sesión). Se realizó una colonoscopia que mostró mucosa del ciego con múltiples ulceraciones planas cubiertas por fibrina. Se tomó una biopsia, en la que se observó un proceso de colitis actínica con cambios más
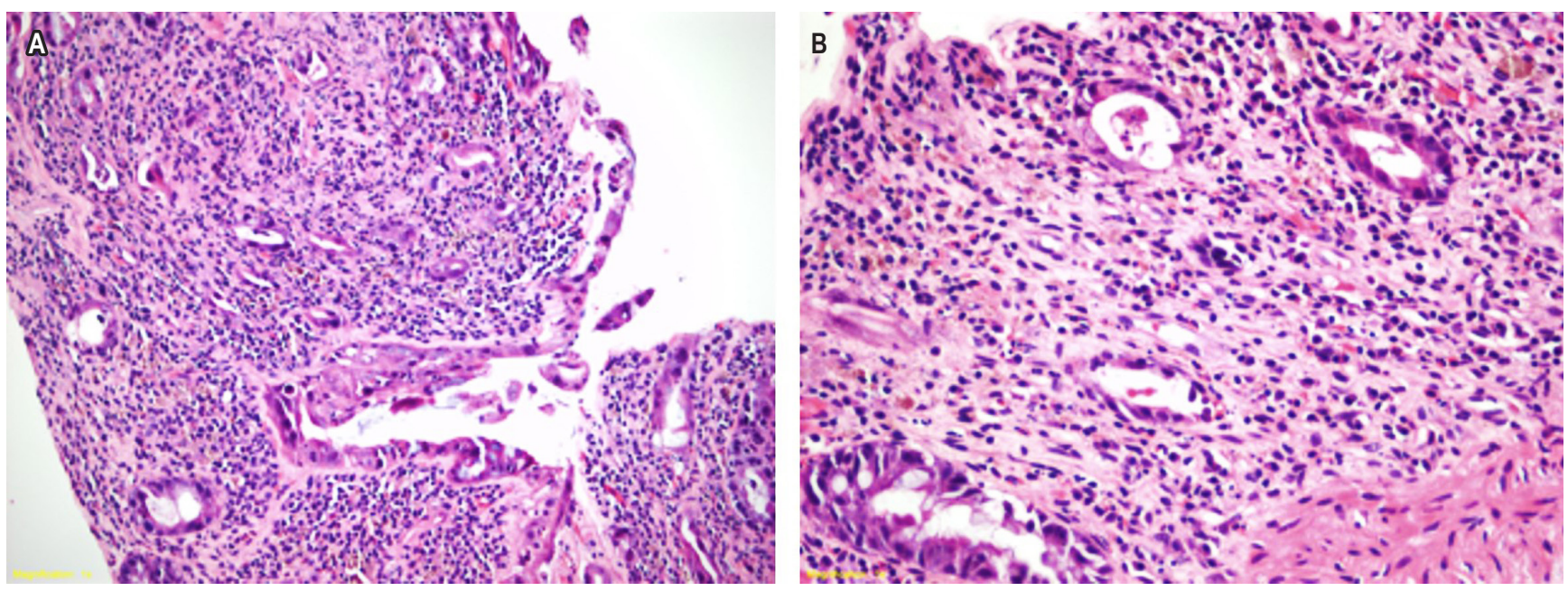

Figura 1. A. HE 4 X, pérdida y miniaturización de glándulas. B. HE 40 X, nucleomegalia con citomegalia. 
graves que en el caso previo, marcada distorsión arquitectural, pérdida y miniaturización de las criptas, formación de microabscesos, estroma fibroso con rico infiltrado inflamatorio polimorfonuclear eosinófilo, fibroblastos y células endoteliales reactivas y cambios regenerativos con células con citomegalia y nucleomegalia sin alteración significativa de la relación núcleo-citoplasma (Figura 2). Con estos hallazgos se diagnosticó una colitis actínica aguda, por lo que el paciente recibió medidas de soporte y tuvo una buena evolución.

\section{DISCUSIÓN}

La radiación ionizante es una causa conocida de enfermedad intestinal (4). Walsh fue quien realizó el primer reporte de daño al intestino inducido por radioterapia, en 1897, solo dos años después del descubrimiento de los rayos $\mathrm{X}$ (5). A pesar de los avances en las técnicas de radioterapia, es frecuente que se presente colitis actínica luego del tratamiento con radioterapia en la región pélvica para el manejo de neoplasias ginecológicas, urológicas y anorrectales. En estos casos se ha reportado que hasta un $50 \%$ de los pacientes reporta síntomas $(3,6)$.

Dentro del abordaje médico de la colitis actínica, la colonoscopia aporta información indispensable que ayuda a dirigir el diagnóstico. Entre los hallazgos se ha descrito la presencia de edema, eritema, friabilidad, úlceras o necrosis de la mucosa de acuerdo con el grado de inflamación (7). En los casos reportados, el primer paciente presentó eritema en la mucosa y el segundo presentó mayor grado de inflamación con presencia de úlceras. Usualmente las biopsias no son necesarias para hacer el diagnóstico, pero como en los casos reportados, es necesaria debido a la alta sospecha del endoscopista y el antecedente de la historia clínica. Adicionalmente, en algunos casos las biopsias pueden ayudar a excluir otras causas de colitis (3). Los diagnósticos diferenciales a considerar incluyen infección, enfermedad inflamatoria intestinal, otras causas de colitis de patrón isquémico, enfermedad diverticular y colitis asociada con medicamentos (8).

En relación con el estudio histopatológico, tanto la fase aguda como la crónica corresponden a un patrón de colitis isquémica aguda y crónica, respectivamente. En la fase aguda, los cambios son más evidentes en el epitelio, con citomegalia, nucleomegalia, picnosis y cariorrexis, abundante actividad mitótica con frecuentes figuras atípicas; hay miniaturización y pérdida de las criptas, pérdida de mucina con infiltrado inflamatorio de la lámina propia con predominio de polimorfonucleares neutrófilos y de células plasmáticas, con formación de abscesos en las criptas. Por el contrario, en la fase crónica, los cambios observados son predominantemente estromales, se observa un tejido conectivo hialinizado que ocupa la lámina propia, atipia reactiva en fibroblastos y células endoteliales, obliteración y hialinización de vasos sanguíneos, y atrofia de la muscular propia (8).

En nuestros pacientes, la historia clínica, junto con los hallazgos endoscópicos e histopatológicos, permitió orientar el diagnóstico hacia una colitis actínica aguda.
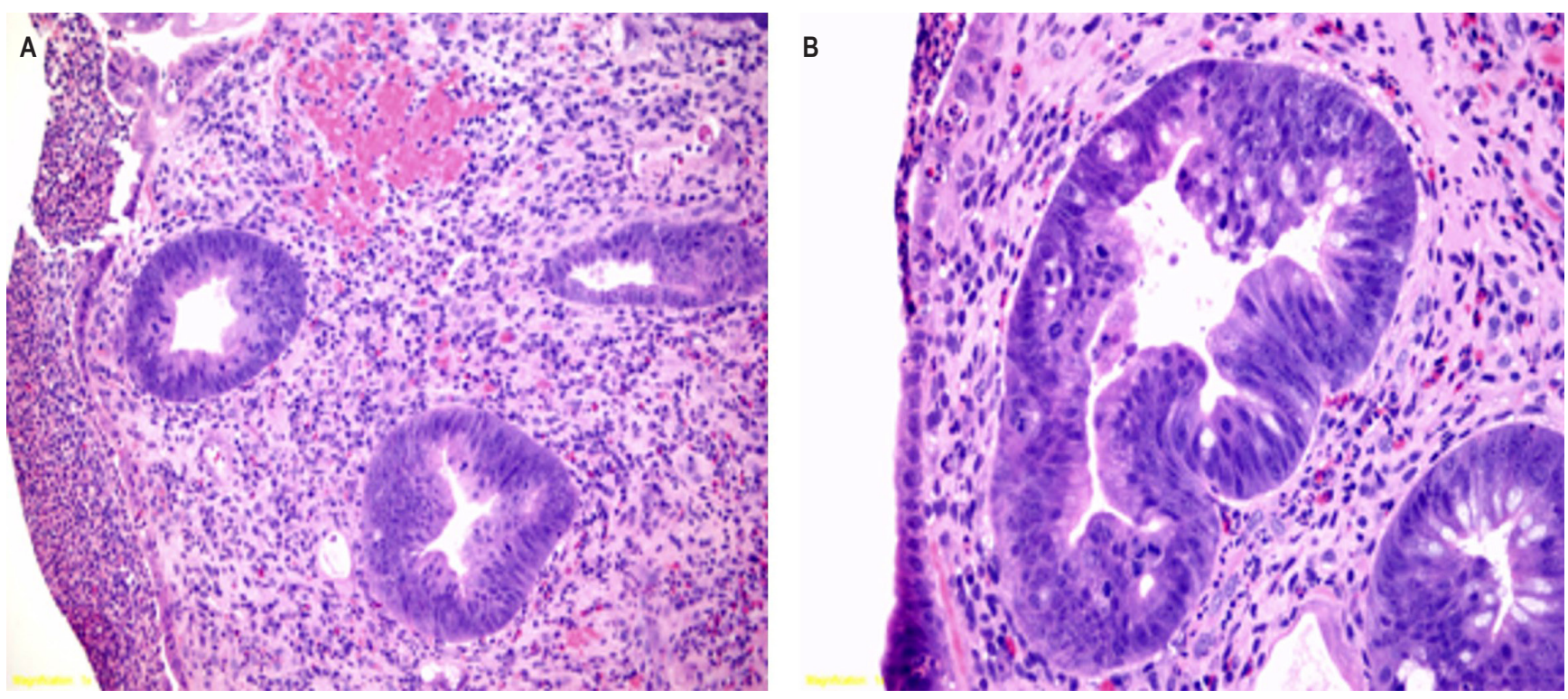

Figura 2. A. HE 4 X, distorsión arquitectural con pérdida y miniaturización de las criptas. B. HE 40 X, estroma fibroso con abundantes eosinófilos. 
A pesar de ser el recto la localización más frecuentemente reportada, el ciego y el íleon terminal, al ser de localización pélvica, se convierten en un factor de riesgo para que estos segmentos anatómicos sean órganos en los cuales se aumente la probabilidad del impacto directo de la radioterapia, Igualmente, es importante destacar que la enfermedad en la fase aguda suele ser autolimitada y resolverse con medidas de soporte (9), tal y como se observó en los dos casos presentados. Por el contrario, en la fase crónica el manejo es más complejo debido a la evolución progresiva de la enfermedad que incluye fibrosis, edema, endarteritis, obstrucción, perforación y malignidad, por lo que se usan diferentes tratamientos como el oxígeno hiperbárico, la cauterización endoscópica y la cirugía, de acuerdo con cada caso (10).

\section{CONCLUSIÓN}

La colitis actínica de localización cecal es una condición infrecuente. Nuestros casos corresponden a los primeros reportados en esa localización, ambos asociados con radiación dirigida a metástasis pélvicas. El personal involucrado en el cuidado de estos pacientes debe considerar la colitis por radioterapia como un diagnóstico diferencial en pacientes que presentan sangrado gastrointestinal y que han sido irradiados como parte de su tratamiento de neoplasias primarias o metastásicas. El conocimiento de esta entidad conduce a un diagnóstico oportuno, previene complicaciones y disminuye la morbilidad a corto y largo plazo de estos pacientes.

\section{REFERENCIAS}

1. Qadeer M, Vargo J. Approaches to the prevention and management of radiation colitis. Curr Gastroenterol Rep. 2008;10(5):507-13. https://doi.org/10.1007/s11894-008-0093-9

2. Kennedy, G. Heise C. Radiation colitis and proctitis. Clin Colon Rectal Surg. 2007;20(1):64-72. https://doi.org/10.1055/s-2007-970202

3. Sarin A, Safar B. Management of radiation proctitis. Gastroenterol Clin N Am. 2013;42(4):913-25. https://doi.org/10.1016/j.gtc.2013.08.004

4. Leupin N, Curschmann J, Kranzbühler H, Maurer CA, Laissue JA, Mazzucchelli L. Acute radiation colitis in patients treated with short-term preoperative radiotherapy for rectal cancer. Am J Surg Pathol. 2002;26(4):498-504. https://doi.org/10.1097/00000478-200204000-00013

5. Theis VS, Sripadam R, Ramani V, Lal S. Chronic radiation enteritis. Clin Oncol (R Coll Radiol). 2010;22(1):70-83. https://doi.org/10.1016/j.clon.2009.10.003
6. Sinkó, D, Baranyai Z, Nemeskéri C, Teknos D, Jósa V, Hegedus L MA. Symptoms, diagnosis and treatment of radiation-induced enteritis Orv Hetil. 2010;151(36):1450-4. https://doi.org/10.1556/OH.2010.28939

7. Kennedy GD, Heise CP. Radiation colitis and proctitis. Clin Colon Rectal Surg. 2007;20:64-72. https://doi.org/10.1055/s-2007-970202.

8. Mollà M, Biete A, Piqué J, Panés J. Lesiones intestinales de la radioterapia. Gastroenterol Hepatol. 2001;24(9):454-60. https://doi.org/10.1016/S0210-5705(01)79002-4

9. Peter R McNally. GI/Liver Plus, Secrets Plus. Colitis: pseudomembranous, microscopic, and radiation. 4.a edición. Elsevier; 2010. p. 349-54. https://doi.org/10.1016/B9780-323-06397-5.00050-2.

10. Kountouras J, Zavos C. Recent advances in the management of radiation colitis. World J Gastroenterol. 2008; 14(48):7289-301. https://doi.org/10.3748/wjg.14.7289 